\title{
MULTI-CLASS DECISION PROCEDURES FOR THE INSPECTION OPERATION
}

\author{
MAJID JARAIEDI \\ Department of Industrial Engineering, \\ West Virginia University, Morgantown, WV 26506 \\ RICHARD S. SEGALL \\ Department of Economics \& Decision Sciences, \\ Arkansas State University, State University, AR 72467 \\ VENKATRAMANI RAJAMOHAN \\ Department of Industrial Engineering, \\ West Virginia University, Morgantown, WV 26506
}

\begin{abstract}
Increasing the amount of inspection activities and inspecting a large fraction of the items are two approaches that are used to improve products' quality. In this paper, as in a precedent article by Jaraiedi, et al.[5], 100\% inspection in combination with multiple-criteria decision (MCD) response is considered. Three different inspection procedures for the multi-stage inspection are presented. Performance of these three procedures are derived and the Average Outgoing Quality (AOQ), Average False Rejected (AFR), and Overall Average Fraction Inspected (SAFI) for all procedures are compared. Two examples are discussed in depth to illustrate numerical comparisons of these procedures.
\end{abstract}

Keywords: Quality, Multi-Criteria Inspection, AOQ, AFR, SAFI

\section{Introduction}

Quality improvement is regarded as one of the key points affecting both the consumers' purchasing decisions and a firm's competitive position. Every process in a production system eventually influences the product's quality and an inspection function is a key operation in any production process. Due to reduction in the costs of inspection systems and the increasing requirements of the marketplace, the use of $100 \%$ inspection at one or more stages of a manufacturing process is now economically viable in many instances. In many manufacturing processes, $100 \%$ inspection is also becoming increasingly important to the detection of moderate shifts in the performance of a process. An example of such a process is the manufacture of integrated circuits, as remarked in Pesotchinsky [8]. Inspection may be done on a sample taken from a lot or on the entire lot. Sampling schemes are established to provide the manufacturer and the customer an acceptable quality level. However, some researchers have made a strong argument against the use of sampling inspection. A policy of zero or $100 \%$ inspection was recommended, when the process quality remains at a stable level, by Deming [3]. Ott [7]gave an exam- 
Table 1: Multi-Class Decision Outcome Matrix

\begin{tabular}{|c|l|l|}
\hline \multicolumn{1}{|c|}{ DECISIONS } & PRODUCTS CONFORMING & PRODUCTS NONCONFORMING \\
\hline ACCEPT SURE & $P($ NO ERROR) $=1-\alpha 1-\alpha 2-\alpha 3$ & $P($ accept sure $)=\beta 1$ \\
\hline ACCEPT NOT SURE & $P($ accept not sure $)=\alpha 3$ & $P($ accept not sure $)=\beta 2$ \\
\hline REJECT NOT SURE & $P($ reject not sure $)=\alpha 2$ & $P($ reject not sure $)=\beta 3$ \\
\hline REJECT SURE & $P($ reject sure $)=\alpha 1$ & $P($ NO ERROR $)=1-\beta 1-\beta 2-\beta 3$ \\
\hline
\end{tabular}

ple for multiple $100 \%$ inspection of a TV component. One method of improving the outgoing quality is to subject the lot to repeated or multiple inspections such that the few nonconforming items that might have escaped detection at the first inspection would be caught during the subsequent inspections. Unfortunately, this also tends to increase the cost of the inspection operation, but it can maintain the product quality at an acceptable level. Beainy and Case [2] presented the AOQ for both single and double sampling inspection, with perfect inspection as well as error-prone inspection. Multi-class decision inspection is based on the realization that the inspectors usually have to make a decision on how to classify the product even if they are not sure whether or not it is conforming. They need other response categories to describe their unsure judgement and make further inspections on those items. In most cases, inspection may not result in rigid classification of items into rejected or accepted categories; it is more reasonable to interpret inspector behaviour by using a rating method, as described by Green and Swets [4]. Contrary to the binary decision method where the inspector is allowed only two responses (accept/reject), the rating method allows any number of responses. A three-class procedure for acceptance sampling plans by variables was presented by Newcombe and Allen[6]. Baker [1] used the rating method to analyse the performance of single inspection process; he devised four categories for the responses given by the inspectors. They are "Accept-Sure", "Accept-Not Sure", "Reject-Not Sure", and "Reject-Sure". Eight possible outcomes associated with this multi-class decision are shown in Table 1. 


\subsection{Measuring the Performance of Inspection}

A perfect inspection model is one in which the inspector never makes a mistake. For instance, given an inspection activity consisting of 100 items in which five are nonconforming, not only does the inspector find the five, he/she also classifies the remaining 95 as conforming items. Given this assumption, the modelling problem reduces to that of deriving the best quality control policy. Average Outgoing Quality (AOQ), and Average Fraction Inspected (AFI) are two common performance measures of an inspection operation, and Average False Rejected (AFR) is a relatively new measure of an inspection operation. The AOQ and AFR depend upon several factors, prominent among which is the inspector's decision mechanism to accept or reject an item. AFI for single sampling inspection is basically the ratio of the number of items that are inspected to the number of items produced in the long run. While $A O Q$ is a measure of the desirable outgoing quality level, AFR is employed to measure reworked or scraped cost of the rejected items. AFI is employed to measure time/cost efficiency of the inspection method. The definition and features of these three measures are presented below. Average Outgoing Quality (AOQ) gives the expected fraction of nonconforming items in the accepted lots as a function of incoming quality level, and, thus, is the most common indicator of the outgoing quality level. It can be computed as:

$$
A O Q=\frac{\text { Expectednumberofnonconformingitemswhichwereundetected }}{\text { Totalnumberofitemswhichwereaccepted }}
$$

The AOQ measure has a numerical range of values from zero to unity. The Average False Rejected (AFR) is based upon the second measure of Wallack and Adams[10]. AFR gives the expected fraction of conforming items in the rejected lots as a function of wasted, reworked or scrapped cost. It may be defined as:

$$
A F R=\frac{\text { Expectednumberofconformingitemswhichwererejected }}{\text { Totalnumberofitemswhichwererejected }}
$$

The AFR measure has the same numerical range as AOQ which is from one to unity. Average Fraction Inspected (AFI) gives the expected fraction of inspected items to the number of products in the lot as a function of incoming quality, and, thus, is the most common indicator of the inspection efficiency. That is:

$$
A F I=\frac{\text { Expectednumberofitemswhichwereinspected }}{\text { Totalnumberofitemsatthebeginning }}
$$

\section{Multi-Class Inspection Procedures}

Consider a multi-stage inspection scheme. In the first stage, the inspector classifies all items (100\% inspection) into the four categories devised by Baker[1]. During the next stage, the items in one or more categories from the preceding stage are reinspected and classified into these four categories again. Inspection is repeated until 
Table2: Multi-Class Decision in the Multi-stage Inspection Procedures

\begin{tabular}{|l|l|l|l|l|}
\hline & Accept Sure & Accept Not Sure & Reject Not Sure & Reject Sure \\
\hline PROCEDURE 1 & Reinspection & \multicolumn{2}{|c|}{ Reject } & Reject \\
\hline PROCEDURE 2 & Reinspection & Reject \\
\hline PROCEDURE 3 & Reinspection & \\
\hline
\end{tabular}

the desirable quality level is reached. In this paper, three inspection procedures are considered, as summarized in Table 2.

The expressions for AOQ, AFR, AFI, and SAFI of these three procedures in each stage are derived in the following sections. The reader is referred to Tsai [9]for a complete derivations of the expressions for AOQ, AFR, AFI, and SAFI for each procedure. Note that these expressions can be written in the form of a multiple of $\mathrm{P}$ and/or (1-P) and one or two constants that are functions of the inputs for $\alpha$ and $\beta$ at each stage. Here, $\mathrm{P}$ is the probability of an item being nonconforming. The difference between the three procedures is mainly based on the values of these constants.

\subsection{Symbols and Notations}

Symbols and notations used for various derivations are summarized below.

$\alpha_{i 1}=$ probability that a conforming item is classified as "Reject-Sure" in stage $\mathrm{i}$

$\alpha_{i 2}=$ probability that a conforming item is classified as "Reject-Not Sure" in stage i

$\alpha_{i 3}=$ probability that a conforming item is classified as "Accept-Not Sure" in stage i

$\beta_{i 1}=$ probability that a nonconforming item is classified as "Accept-Sure" in stage i

$\beta_{i 2}=$ probability that a nonconforming item is classified as "Accept-Not Sure" in stage i

$\beta_{i 3}=$ probability that a nonconforming item is classified as "Reject-Not Sure" in stage i

$\left(A S_{c}\right)_{i}=$ expected number of conforming items classified as "Accept-Sure" in stage i

$\left(A N_{c}\right)_{i}=$ expected number of conforming items classified as "Accept-Not Sure" in stage $\mathbf{i}$ 
$\left(R N_{c}\right)_{i}=$ expected number of conforming items classified as "Reject-Not Sure" in stage i

$\left(R S_{c}\right)_{i}=$ expected number of conforming items classified as "Reject-Sure" in stage $\mathrm{i}$

$\left(A S_{n}\right)_{i}=$ expected number of nonconforming items classified as "Accept-Sure" in stage i

$\left(A N_{n}\right)_{i}=$ expected number of nonconforming items classified as "Accept-Not Sure" in stage $\mathrm{i}$

$\left(R N_{n}\right)_{i}=$ expected number of nonconforming items classified as "Reject-Not Sure" in stage $\mathrm{i}$

$\left(R S_{n}\right)_{i}=$ expected number of nonconforming items classified as "Reject-Sure" in stage $\mathrm{i}$

\subsection{Procedure 1}

The items in the "Accept-Sure" category from preceding stage will be reinspected in the next stage, other items are regarded as rejected. The inspection process of Procedure 1 and the corresponding probabilities are shown in Figure 1. The probability of an item being nonconforming is $\mathrm{P}$, while the probability of being conforming is 1 - P. Expressions for the AOQ, AFR, AFI, and SAFI in stage $\mathrm{k}$ are derived as follows. Expected number of conforming items classified into "AcceptSure",

$$
\left(A S_{c}\right)_{k}=N(1-P) \prod_{i=1}^{k}\left(1-\alpha_{i 1}-\alpha_{i 2}-\alpha_{i 3}\right)
$$

Expected number of conforming items classified into "Accept-Not Sure",

$$
\left(A N_{c}\right)_{k}=N(1-P) \alpha_{k 3} \prod_{i=1}^{k-1}\left(1-\alpha_{i 1}-\alpha_{i 2}-\alpha_{i 3}\right)
$$

Expected number of conforming items classified into "Reject-Not Sure",

$$
\left(R N_{c}\right)_{k}=N(1-P) \alpha_{k 2} \prod_{i=1}^{k-1}\left(1-\alpha_{i 1}-\alpha_{i 2}-\alpha_{i 3}\right)
$$

Expected number of conforming items classified into "Reject-Sure",

$$
\left(R S_{c}\right)_{k}=N(1-P) \alpha_{k 1} \prod_{i=1}^{k-1}\left(1-\alpha_{i 1}-\alpha_{i 2}-\alpha_{i 3}\right)
$$


Figure 1: Inspection Procedure 1

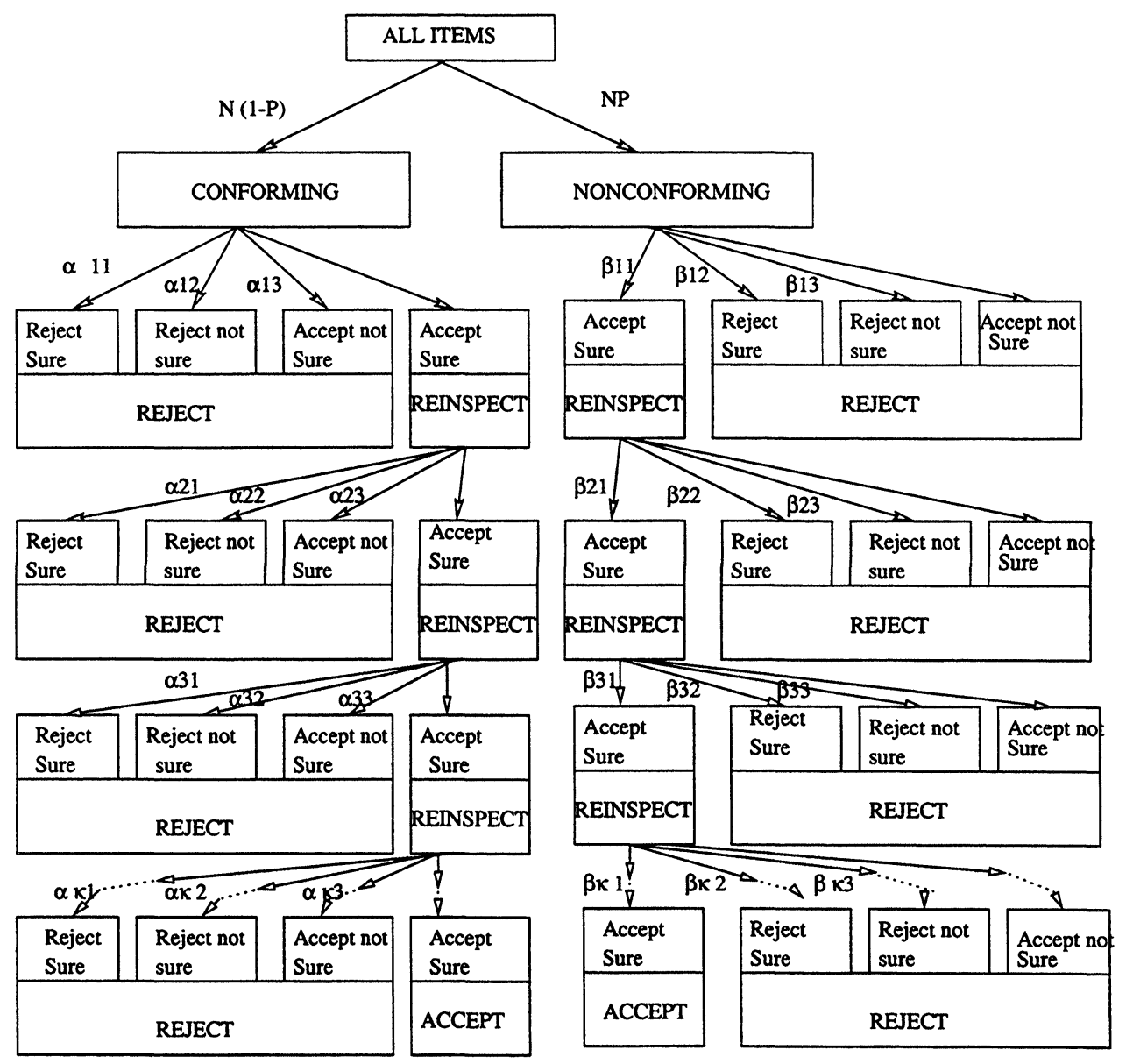


Expected number of nonconforming items classified into "Accept-Sure",

$$
\left(A S_{n}\right)_{k}=N P \prod_{i=1}^{k} \beta_{i 1}
$$

Expected number of nonconforming items classified into "Accept-Not Sure",

$$
\left(A N_{n}\right)_{k}=N P \beta_{k 2} \prod_{i=1}^{k-1} \beta_{i 1}
$$

Expected number of nonconforming items classified into "Reject-Not Sure",

$$
\left(R N_{n}\right)_{k}=N P \beta_{k 3} \prod_{i=1}^{k-1} \beta_{i 1}
$$

Expected number of nonconforming items classified into "Reject-Sure",

$$
\left(R S_{n}\right)_{k}=N P\left(1-\beta_{k 1}-\beta_{k 2}-\beta_{k 3}\right) \prod_{i=1}^{k-1} \beta_{i 1}
$$

The expression for SAFI is:

$$
\begin{aligned}
(S A F I)_{k}= & (A F I)_{1}+(A F I)_{2}+(A F I)_{3} \\
& +\ldots+(A F I)_{k} \\
= & 1+(1-P) \sum_{j=1}^{k-1} \prod_{i=1}^{j}\left(1-\alpha_{i 1}-\alpha_{i 2}-\alpha_{i 3}\right) \\
& +P \sum_{j=1}^{k-1} \prod_{i=1}^{j} \beta_{i 1}
\end{aligned}
$$

Expressions for AOQ, AFR, and AFI are as follows.

$$
\begin{aligned}
(A O Q)_{k} & =\frac{\left(A S_{n}\right)_{k}}{\left.\left(A S_{c}\right)_{k}+\left(A S_{n}\right)_{k}\right)} \\
& =\frac{P \prod_{i=1}^{k} \beta_{i 1}}{(1-P) \prod_{i=1}^{k}\left(1-\alpha_{i 1}-\alpha_{i 2}-\alpha_{i 3}\right)+P \prod_{i=1}^{k} \beta_{i 1}} \\
& =\frac{1}{\frac{1-P}{P}\left[\frac{\prod_{i=1}^{k}\left(1-\alpha_{i 1}-\alpha_{i 2}-\alpha_{i 3}\right)}{\prod_{i=1}^{k} \beta_{i 1}}\right]+1} \\
& =\frac{1}{\frac{1-P}{P} *(\text { Constant })+1}
\end{aligned}
$$




$$
(A F R)_{k}=\frac{1}{\frac{P}{1-P}(\text { AConstant })+1}
$$

where the constant in this formula is

$$
\begin{aligned}
& \left(\left(1-\beta_{11}\right)+\sum_{j=2}^{k} \prod_{i=1}^{j-1} \beta_{i 1}\left(1-\beta_{j 1}\right)\right) /\left[\left(\alpha_{11}+\alpha_{12}+\alpha_{13}\right)\right. \\
& +\sum_{j=2}^{k} \prod_{i=1}^{j-1}\left(1-\alpha_{i 1}-\alpha_{i 2}-\alpha_{i 3}\right)\left(\alpha_{j 1}+\alpha_{j 2}+\alpha_{j 3}\right) \\
& (A F I)_{k}=\frac{\left(A S_{c}\right)_{k-1}+\left(A S_{n}\right)_{k-1}}{N} \\
& =(1-P) \prod_{i=1}^{k-1}\left(1-\alpha_{i 1}-\alpha_{i 2}-\alpha_{i 3}\right)+P \prod_{i=1}^{k-1} \beta_{i 1} \\
& =(1-P) \star(\text { Constant } 1)+P \star(\text { Constant } 2)
\end{aligned}
$$

\subsection{Procedure 2}

The items in the "Reject-Sure" category are considered rejected, other items will be reinspected in the next stage. The inspection process of Procedure 2 is shown in Figure 2.

AOQ, AFR, AFI, and SAFI expressions in stage $\mathrm{k}$ are as follows.

$$
\begin{aligned}
& \left(A S_{c}\right)_{k}=N(1-P)\left(1-\alpha_{k 1}-\alpha_{k 2}-\alpha_{k 3}\right) \prod_{i=1}^{k-1}\left(1-\alpha_{i 1}\right) \\
& \left(A N_{c}\right)_{k}=N(1-P) \alpha_{k 3} \prod_{i=1}^{k-1}\left(1-\alpha_{i 1}\right) \\
& \left(R N_{c}\right)_{k}=N(1-P) \alpha_{k 2} \prod_{i=1}^{k-1}\left(1-\alpha_{i 1}\right) \\
& \left(R S_{c}\right)_{k}=N(1-P) \alpha_{k 1} \prod_{i=1}^{k-1}\left(1-\alpha_{i 1}\right)
\end{aligned}
$$


Figure 2: Inspection Procedure 2

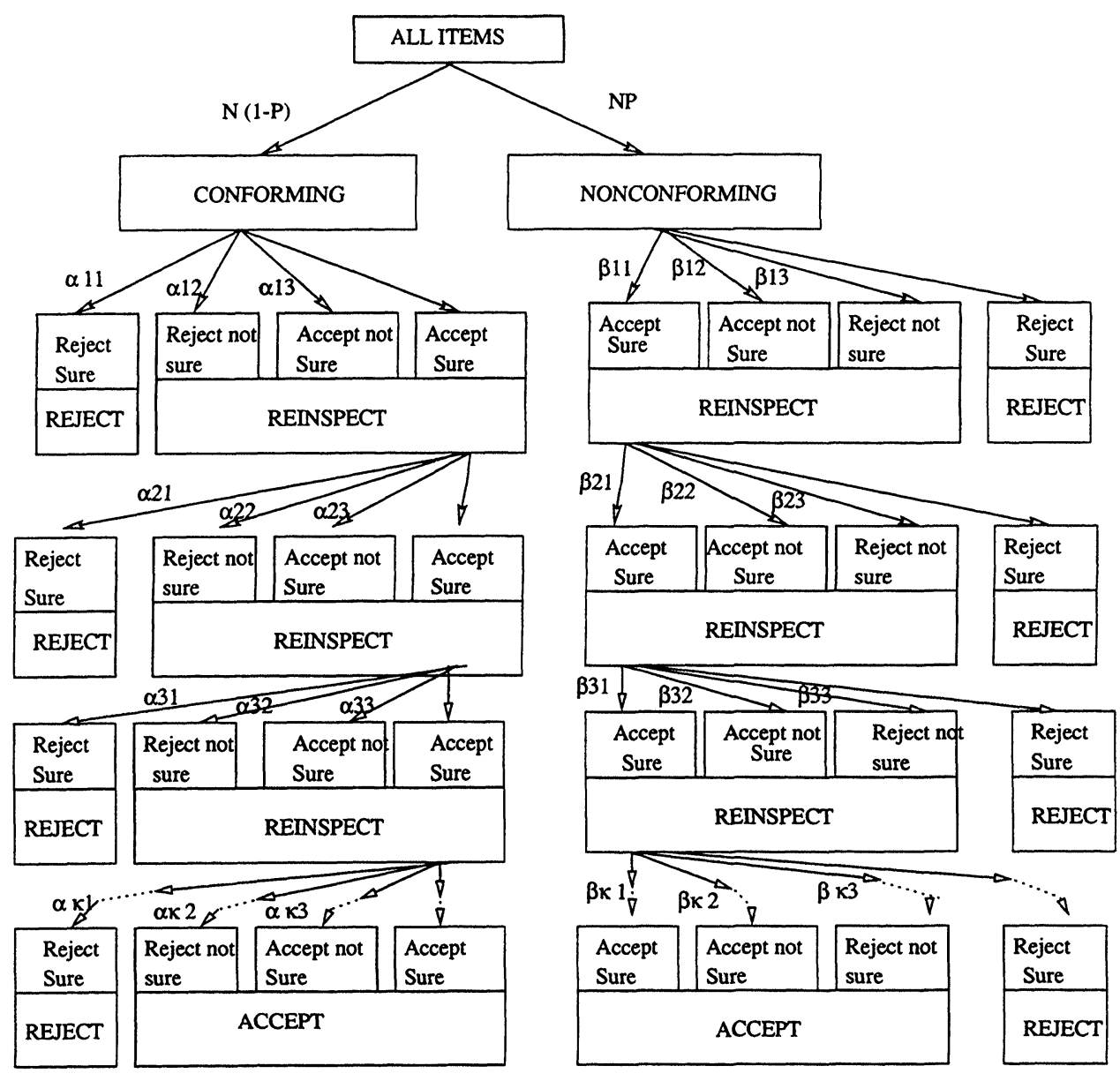




$$
\begin{aligned}
& \left(A S_{n}\right)_{k}=N P \beta_{k 1} \prod_{i=1}^{k-1}\left(\beta_{i 1}+\beta_{i 2}+\beta_{i 3}\right) \\
& \left(A N_{n}\right)_{k}=N P \beta_{k 2} \prod_{i=1}^{k-1}\left(\beta_{i 1}+\beta_{i 2}+\beta_{i 3}\right) \\
& \left(R N_{n}\right)_{k}=N P \beta_{k 3} \prod_{i=1}^{k-1}\left(\beta_{i 1}+\beta_{i 2}+\beta_{i 3}\right) \\
& \left(R S_{n}\right)_{k}=N P\left(1-\beta_{k 1}-\beta_{k 2}-\beta_{k 3}\right) \prod_{i=1}^{k-1}\left(\beta_{i 1}+\beta_{i 2}+\beta_{i 3}\right) \\
& (A O Q)_{k}=\frac{\left(A S_{n}\right)_{k}+\left(A N_{n}\right)_{k}+\left(R N_{n}\right)_{k}}{\left(A S_{c}\right)_{k}+\left(A N_{c}\right)_{k}+\left(R N_{c}\right)_{k}+\left(A S_{n}\right)_{k}+\left(A N_{n}\right)_{k}+\left(R N_{n}\right)_{k}} \\
& =\frac{P \prod_{i=1}^{k}\left(\beta_{i 1}+\beta_{i 2}+\beta_{i 3}\right)}{(1-P) \prod_{i=1}^{k}\left(1-\alpha_{i 1}\right)+P \prod i=1^{k}\left(\beta_{i 1}+\beta_{i 2}+\beta_{i 3}\right)} \\
& =\frac{1}{\frac{1-P}{P}\left[\prod_{i=1}^{k}\left(1-\alpha_{i 1}\right)\right] /\left[\prod_{i=1}^{k}\left(\beta_{i 1}+\beta_{i 2}+\beta_{i 3}\right)\right]+1} \\
& =\frac{1}{\frac{1-P}{P} \star(A \text { Constant })+1} \\
& (A F R)_{k}=\frac{1}{\frac{P}{1-P} \star(\text { AConstant })+1}
\end{aligned}
$$

where the constant in this formula is

$$
\begin{aligned}
\left.\left(1-\beta_{11}-\beta_{12}-\beta_{13}\right)+\sum_{j=2}^{k} \prod_{i=1}^{j-1}\left(\beta_{i 1}+\beta_{i 2}+\beta_{i 3}\right)+\left(1-\beta_{j 1}-\beta_{j 2}-\beta_{j 3}\right)\right] / \\
{\left[\alpha_{11}+\sum_{j=2}^{k} \prod_{i=1}^{j-1}\left(1-\alpha_{i 1}\right) \alpha_{j 1}\right] } \\
(A F I)_{k}=\frac{\left(A S_{c}\right)_{k-1}+\left(A N_{c}\right)_{k-1}+\left(R N_{c}\right)_{k-1}+\left(A S_{n}\right)_{k-1}+\left(A N_{n}\right)_{k-1}+\left(R N_{n}\right)_{k-1}}{N} \\
=(1-P) \prod_{i=1}^{k-1}\left(1-\alpha_{i 1}\right)+P \prod_{i=1}^{k-1}\left(\beta_{i 1}+\beta_{i 2}+\beta_{i 3}\right) \\
=(1-P) \star(\text { Constant } 1)+P \star(\text { Constant } 2)
\end{aligned}
$$




$$
\begin{array}{r}
(S A F I)_{k}=(A F I)_{1}+(A F I)_{2}+(A F I)_{3}+\ldots+(A F I)_{k} \\
=1+\left[(1-P)\left(1-\alpha_{11}\right)+P\left(\beta_{11}+\beta_{12}+\beta_{13}\right)\right] \\
+\ldots+\left[(1-P) \prod_{i=1}^{k-1}\left(1-\alpha_{i 1}\right)+P \prod_{i=1}^{k-1}\left(\beta_{i 1}+\beta_{i 2}+\beta_{i 3}\right)\right] \\
=1+(1-P) \sum_{j=1}^{k-1} \prod_{i=1}^{j}\left(1-\alpha_{i 1}\right)+P \sum_{j=1}^{k-1} \prod_{i=1}^{j}\left(\beta_{i 1}+\beta_{i 2}+\beta_{i 3}\right) \\
=1+(1-P) \star(\text { Constant } 1)+P \star(\text { Constant } 2)
\end{array}
$$

\subsection{Procedure 3}

The items in the "Accept-Sure", and the "Accept-Not Sure" categories from preceding stage will be reinspected in the next stage, other items are considered rejected. If is equal to $1+2$, and is equal to $1+2$, then this procedure will be the same as the traditional binary decision method as previously discussed. The inspection process of Procedure 3 is shown in Figure 3.

$\mathrm{AOQ}, \mathrm{AFR}$, AFI, and SAFI expressions in stage $\mathrm{k}$ are given below.

$$
\begin{aligned}
& \left(A S_{c}\right)_{k}=N(1-P)\left(1-\alpha_{k 1}-\alpha_{k 2}-\alpha_{k 3}\right) \prod_{i=1}^{k-1}\left(1-\alpha_{i 1}-\alpha_{i 2}\right) \\
& \left(A N_{c}\right)_{k}=N(1-P) \alpha_{k 3} \prod_{i=1}^{k-1}\left(1-\alpha_{i 1}-\alpha_{i 2}\right) \\
& \left(R N_{c}\right)_{k}=N(1-P) \alpha_{k 2} \prod_{i=1}^{k-1}\left(1-\alpha_{i 1}-\alpha_{i 2}\right) \\
& \left(R S_{c}\right)_{k}=N(1-P) \alpha_{k 1} \prod_{i=1}^{k-1}\left(1-\alpha_{i 1}-\alpha_{i 2}\right) \\
& \left(A S_{n}\right)_{k}=N P \beta_{k 1} \prod_{i=1}^{k-1}\left(\beta_{i 1}+\beta_{i 2}\right) \\
& \left(A N_{n}\right)_{k}=N P \beta_{k 2} \prod_{i=1}^{k-1}\left(\beta_{i 1}+\beta_{i 2}\right)
\end{aligned}
$$


Figure 3: Inspection Procedure 3

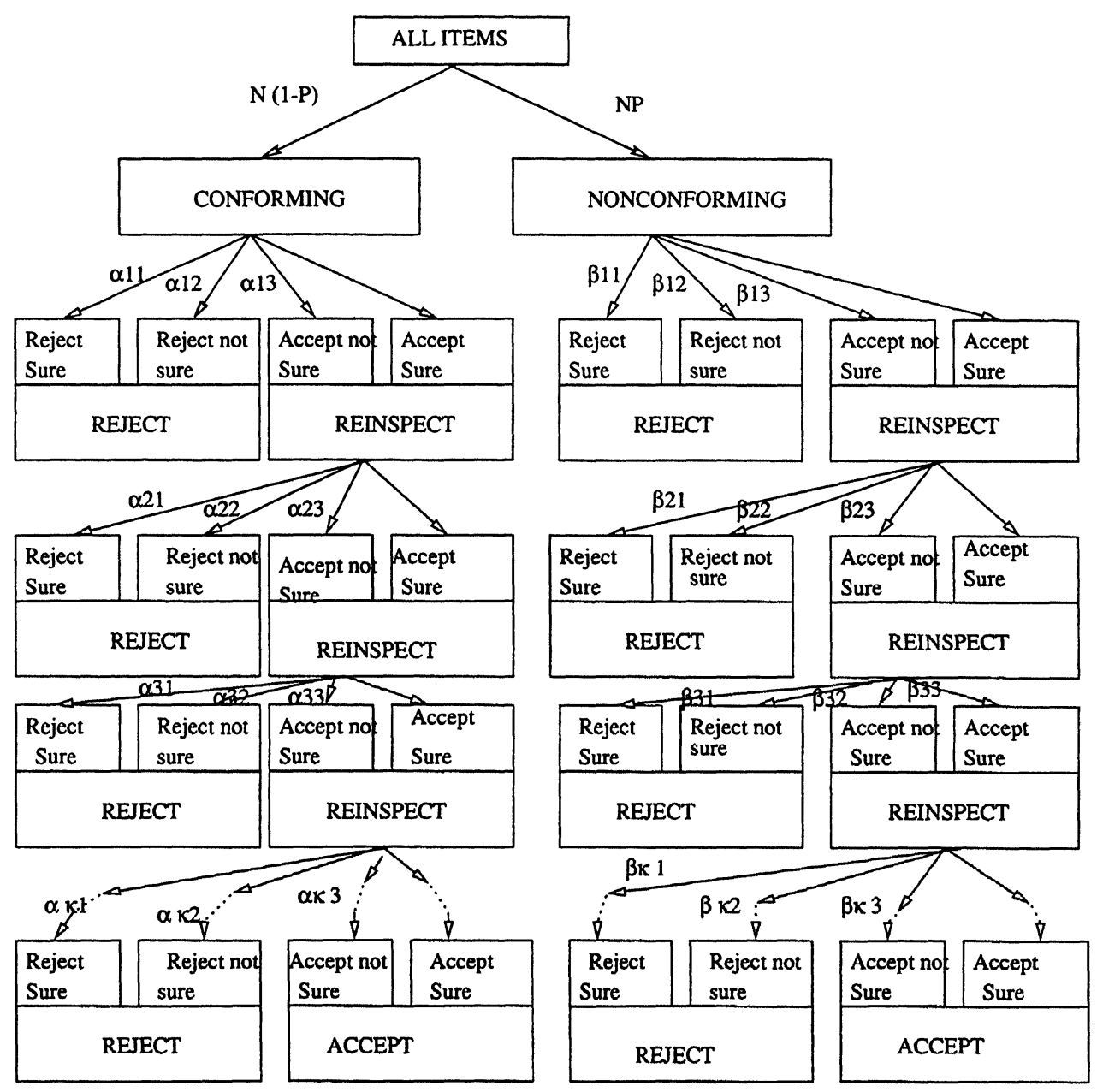




$$
\begin{aligned}
\left(R N_{n}\right)_{k} & =N P \beta_{k 3} \prod_{i=1}^{k-1}\left(\beta_{i 1}+\beta_{i 2}\right) \\
\left(R S_{n}\right)_{k} & =N P\left(1-\beta_{k 1}-\beta_{k 2}-\beta_{k 3}\right) \prod_{i=1}^{k-1}\left(\beta_{i 1}+\beta_{i 2}\right) \\
(A O Q)_{k} & =\frac{\left(A S_{n}\right)_{k}+\left(A N_{n}\right)_{k}}{\left(A S_{c}\right)_{k}+\left(A N_{c}\right)_{k}+\left(A S_{n}\right)_{k}+\left(A N_{n}\right)_{k}} \\
& =\frac{P \prod_{i=1}^{k}\left(\beta_{i 1}+\beta_{i 2}\right)}{(1-P) \prod_{i=1}^{k}\left(1-\alpha_{i 1}-\alpha_{i 2}\right)+P \prod_{i=1}^{k}\left(\beta_{i 1}+\beta_{i 2}\right)} \\
& =\frac{1}{\frac{1-P}{P}\left[\prod_{i=1}^{k}\left(1-\alpha_{i 1}-\alpha_{i 2}\right)\right] /\left[\prod_{i=1}^{k}\left(\beta_{i 1}+\beta_{i 2}\right)\right]+1} \\
& =\frac{1}{\frac{1-P}{P}(A \text { Constant })+1} \\
(A F R)_{k} & =\frac{1}{\frac{P}{1-P}(\text { AConstant })+1}
\end{aligned}
$$

where the constant in the formula is

$$
\begin{gathered}
\left.\left(1-\beta_{11}-\beta_{12}\right)+\sum_{j=2}^{k} \prod_{i=1}^{j-1}\left(\beta_{i 1}+\beta_{i 2}\right)\left(1-\beta_{j 1}-\beta_{j 2}\right)\right] /\left[\left(\alpha_{11}+\alpha_{12}\right)\right. \\
\quad+\sum_{j=2}^{k} \prod_{i=1}^{j-1}\left(1-\alpha_{i 1}-\alpha_{i 2}\right)\left(\alpha_{j 1}+\alpha_{j 2}\right) \\
\begin{aligned}
(A F I)_{k}= & \frac{\left(A S_{c}\right)_{k-1}+\left(A N_{c}\right)_{k-1}+\left(A S_{n}\right)_{k-1}+\left(A N_{n}\right)_{k-1}}{N} \\
= & (1-P) \prod_{i=1}^{k-1}\left(1-\alpha_{i 1}-\alpha_{i 2}\right)+P \prod_{i=1}^{k-1}\left(\beta_{i 1}+\beta_{i 2}\right) \\
= & (1-P) \star(C o n s t a n t 1)+P \star(C o n s t a n t 2)
\end{aligned} \\
(S A F I)_{k}=(A F I)_{1}+(A F I)_{2}+(A F I)_{3}+\ldots+(A F I)_{k} \\
=1+(1-P) \sum_{j=1}^{k-1} \prod_{i=1}^{j}\left(1-\alpha_{i 1}-\alpha_{i 2}\right)
\end{gathered}
$$




$$
\begin{array}{r}
+P \sum_{j=1}^{k-1} \prod_{i=1}^{j}\left(\beta_{i 1}+\beta_{i 2}\right) \\
=1+(1-P) \star(\text { Constant } 1)+P \star(\text { Constant } 2)
\end{array}
$$

\section{Examples}

The following examples are presented to illustrate the use of the three inspection procedures described in this paper. Numerical values of AOQ, AFR, AFI, and SAFI were computed for each of these three procedures using a computer program that can be found in Tsai[9].

\section{Example 1}

Assume that the incoming fraction nonconforming is a constant and a three-stage inspection is needed. The probability of various responses are assumed to be the same at each stage. For $P=0.1, k=3$, let:

$$
\alpha_{i 1}=3 \%, \alpha_{i 2}=6 \%, \alpha_{i 3}=9 \%, \beta_{i 1}=4 \%, \beta_{i 2}=7 \%, \beta_{i 3}=10 \% \text {. }
$$

The AOQ, AFR, and SAFI at the end of the third stage are shown in the Table 3.

Example 2 This example demonstrates the effect of change in the incoming fraction nonconforming on the three indicators. Using the same assumptions as in Example 1, let $k=3$, and $\alpha_{i 1}=3 \%, \alpha_{i 2}=6 \%, \alpha_{i 3}=9 \%, \beta_{i 1}=4 \%, \beta_{i 2}=7 \%$, $\beta_{i 3}=10 \%$. P is changed from $5 \%$ to $25 \%$ in increments of $5 \%$, and the results of calculations for different incoming fractions nonconforming are shown in Table 4. It can be seen that the $A O Q$ increases when the incoming fraction nonconforming is increased. The sharpest increase rate of $A O Q$ is in Procedure 3 and the order of the $\mathrm{AOQ}$ in this example is:

\section{Proc. $1<$ Proc. $3<$ Proc. 2}

The AFR's decrease with an increase in the incoming fraction nonconforming. All three procedures have almost the same decreasing rate of AFR. When $\mathrm{P}$ is decreased from $25 \%$ to $5 \%$, the AFR for the three procedures increases by $32 \%, 42 \%$, and $40 \%$. The order of the AFR in this example is:

$$
\text { Proc. } 2<\text { Proc. } 3<\text { Proc. } 1
$$

When the incoming fraction nonconforming is increased, the SAFI of Procedures 1 , and 2 and 3 are decreased. The effect of the increase in the incoming fraction nonconforming is significant for Procedures 1, and 2. The order of the SAFI in this example is:

$$
\text { Proc. } 1<\text { Proc. } 3<\text { Proc. } 2
$$


Table 3: AOQ, AFR and SAFI of Example 1

\begin{tabular}{|c|c|c|c|}
\hline \multicolumn{5}{|c|}{ AOQ (\%) } \\
\hline STAGE & PROC. 1 & PROC. 2 & PROC. 3 \\
\hline 1 & .5391 & 2.3490 & 1.3253 \\
\hline 2 & .0264 & .5181 & .1621 \\
\hline 3 & .0013 & .1126 & .0196 \\
\hline \multicolumn{5}{|c|}{ AFR (\%) } \\
\hline 1 & 62.79 & 25.47 & 47.64 \\
\hline 2 & 74.70 & 35.77 & 61.02 \\
\hline 3 & 80.15 & 44.24 & 68.95 \\
\hline \multicolumn{5}{|c|}{ SAFI } \\
\hline 1 & 1 & 1 & 1 \\
\hline 2 & 1.74 & 1.89 & 1.83 \\
\hline 3 & 2.35 & 2.75 & 2.57 \\
\hline
\end{tabular}


Table4: AOQ, AFR, and SAFI of Example 1

\begin{tabular}{|c|c|c|c|}
\hline \multicolumn{4}{|c|}{$\mathrm{AOQ}(\%)$} \\
\hline P & Proc. 1 & Proc. 2 & Proc. 3 \\
\hline $5 \%$ & .006 & .534 & .009295 \\
\hline $10 \%$ & .0013 & .1126 & .0196 \\
\hline $15 \%$ & .0020 & 1718 & .03116 \\
\hline $20 \%$ & .0029 & .2530 & .044137 \\
\hline $25 \%$ & .0039 & .3371 & .58841 \\
\hline \multicolumn{4}{|c|}{ AFR (\%) } \\
\hline $5 \%$ & 89.50 & 62.61 & 82.42 \\
\hline $10 \%$ & 80.15 & 44.24 & 68.95 \\
\hline $15 \%$ & 71.77 & 33.31 & 58.30 \\
\hline $20 \%$ & 64.22 & 26.07 & 49.67 \\
\hline $25 \%$ & 57.38 & 20.19 & 42.53 \\
\hline \multicolumn{4}{|c|}{ SAFI } \\
\hline $5 \%$ & 242 & 2.83 & 263 \\
\hline $10 \%$ & 2.35 & 2.75 & 2.55 \\
\hline $15 \%$ & 227 & 2.66 & 2.47 \\
\hline $20 \%$ & 220 & 2.58 & 239 \\
\hline $25 \%$ & 2.13 & 2.50 & 2.32 \\
\hline
\end{tabular}




\section{CONCLUSIONS AND FUTURE DIRECTIONS OF RESEARCH}

The main purpose of multi-stage inspection is to achieve a higher outgoing quality in the long- run. Obviously, the cost of additional inspection must be carefully balanced against the benefit of improved outgoing quality. Given the assumptions made in this research, it is possible to evaluate the improvement in AOQ after each stage. This gain in quality, then, must be compared to the cost of hiring an additional inspector for the extra stage. In general, AOQ must be balanced against AFR and SAFI which indicate the cost of acceptable items falsely rejected and the total inspection effort, respectively. This comparison must be made for all the six procedures addressed in this research. For instance, in Example 2 for $P=5 \%$ after 3 stages of inspection Procedures 2 and 3 may be compared to select the one that is more cost effective. Procedure 3 achieves a much lower AOQ compared to Procedure 2. However, this improvement is accompanied with 1.31 times more in the cost of good items rejected, but the amount of inspection is roughly the same. If the $0.009 \%$ outgoing quality achieved by Procedure 3 does justify the expenditure of additional resources, then, this procedure will be chosen over Procedure 2. The future directions of the research should include allowing more categories of inspection response, and a mix of reinspection policies at selected stages as described in our earlier paper. Allowing five or more categories of inspector response for the three new procedures presented in this paper will make it possible to devise a variety of reinspection policies that may result in improved overall quality and lower costs in the long run. A mix of reinspection policies at various stages should be considered rather than assuming constant reinspection policy at every stage. This would require development of new expressions for AOQ, AFR, and SAFI.

\section{References}

1. E. M. Baker. Signal Detection Theory Analysis of Quality Control Inspector Performance. Journal of Quality Technology, 7:62-71, 1975.

2. I. Beainy and K. E. Case. A Wide Variety of AOQ and ATI Performance Measures with and without Inspection Error. Journal of Quality Technology, 13:1-9, 1981.

3. E. W. Deming. Quality, Productivity, and Competitive Position. MIT Press, Cambridge, MA., 1982.

4. D. M. Green and J. A. Swets. Signal Detection Theory and Psychophysics. John Wiley and Sons,New York, 1964.

5. M. Jaraiedi, R. S. Segall and Y. Tsai. Multi-Criteria Decision Procedures for the Inspection Operation. Journal of Design and Manufacturing, 5: 25-44, 1995.

6. P. A. Newcombe and O. B. Allen. A Three-Class Procedure for Acceptance Sampling by Variables. Technometrics, 30: 415-421, 1988.

7. E. R. Ott. Process Quality Control. McGraw-Hill Book Company, New York, 1975.

8. L. Pesotchinsky. Plans for Very Low Fraction Nonconforming. Journal of Quality Technology, 19:191-196, 1987.

9. Y. Tsai. Multi-Criteria Decision Procedures for the Inspection Operation. M.S. Thesis, Department of Industrial Engineering, West Virginia University, Morgantown, WV, 1993.

10. P. M. Wallack and S. K. Adams. A Comparison of Inspector Performance Measures. AIIE Transactions, 2:97-105, 1970. 


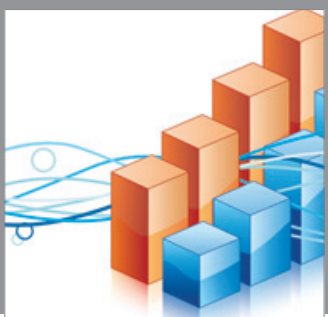

Advances in

Operations Research

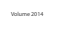

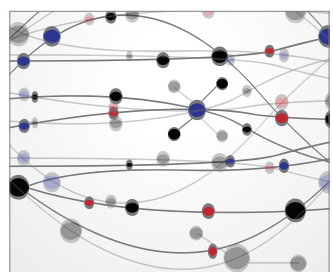

\section{The Scientific} World Journal
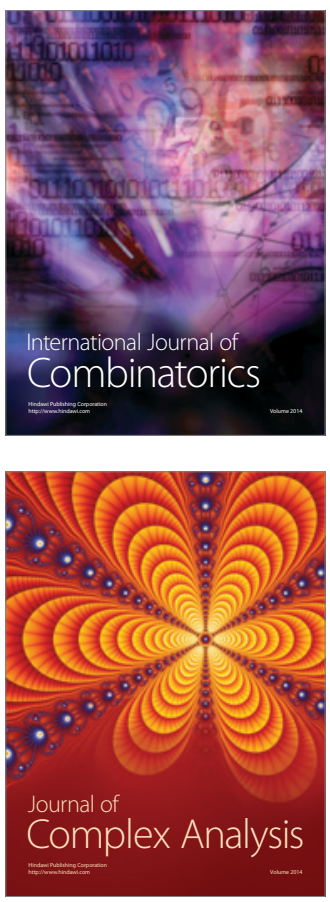

International Journal of

Mathematics and

Mathematical

Sciences
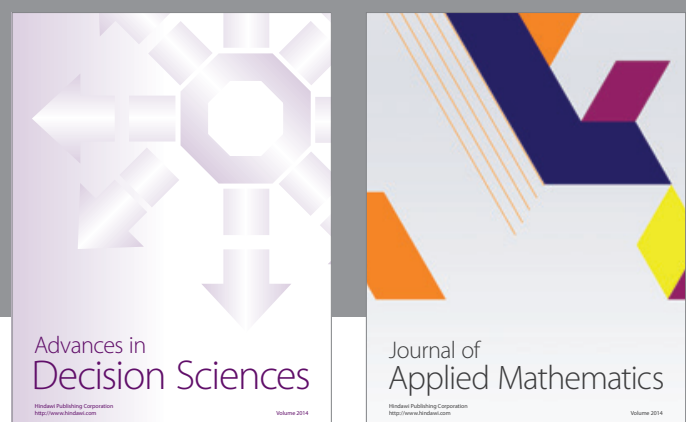

Journal of

Applied Mathematics
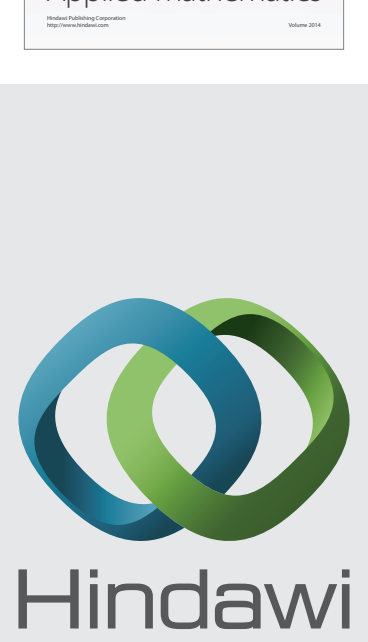

Submit your manuscripts at http://www.hindawi.com
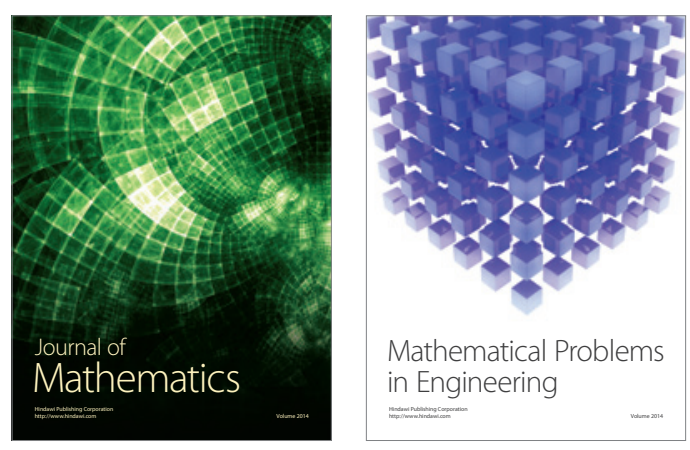

Mathematical Problems in Engineering
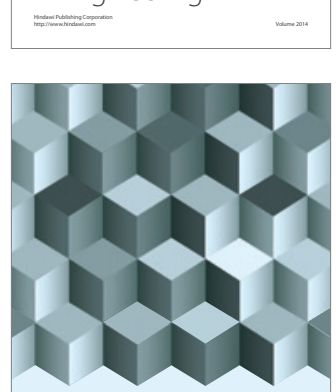

Journal of

Function Spaces
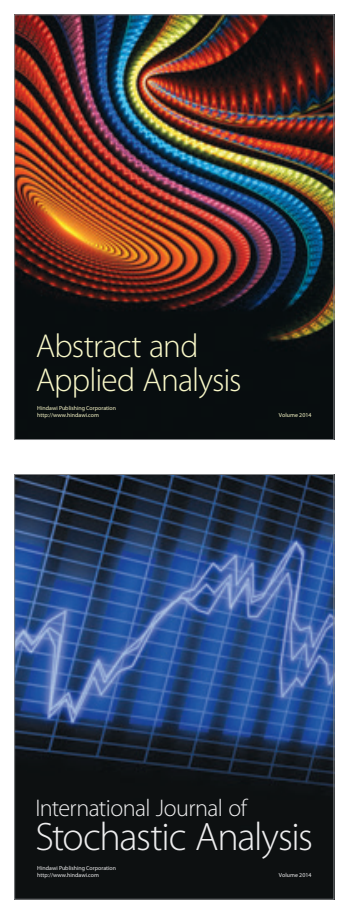

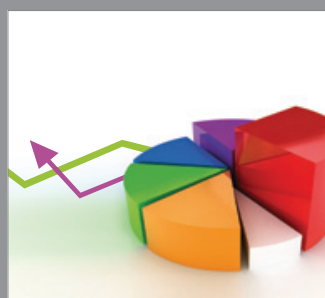

ournal of

Probability and Statistics

Promensencen
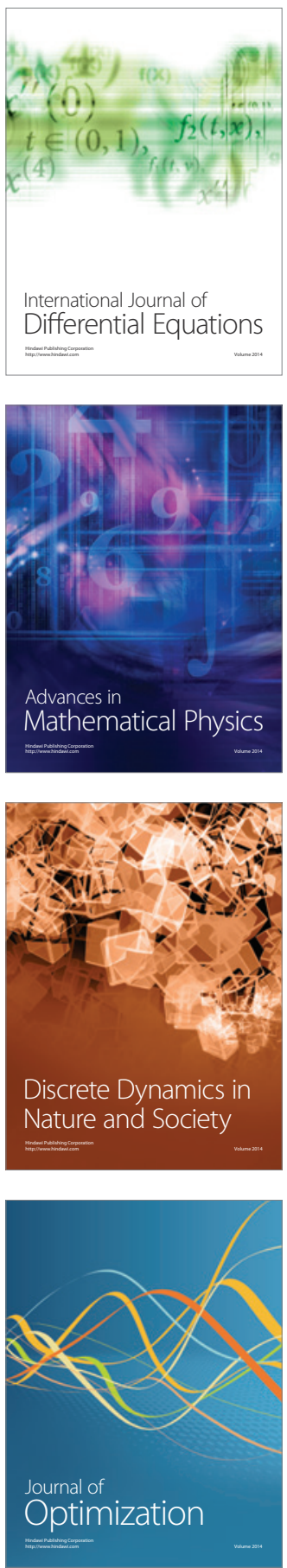